\title{
Performance Comparison of Different Variable Filters for Noise Cancellation in Real-Time Environment
}

\author{
B. Ananda Krishna ${ }^{1}$ and G. V. P. Chandra Sekhar Yadav ${ }^{2}$ \\ ${ }^{1}$ Professor, Department of ECE, Chalapathi Institute of Engineering \& \\ Technology, Guntur, India \\ anand_bk@rediffmail.com, Mobile: 8099970989 \\ ${ }^{2}$ Assistant Professor, Department of ECE, DVR \& Dr. HS MIC College of \\ Technology, Kanchikacherla, Krishna District, Andhra Pradesh, India \\ sekhar.yadav2008@gmail.com, Mobile:9032259527
}

\begin{abstract}
Elimination of noise from the signal is the major task in signal processing applications. Wiener filter removes noise efficiently but it requires large number of computations and it was updated with speed issue with adaptive filter. Adaptive filter has several algorithms to remove noise from the signal. This paper performs cancellation of noise from the signal using wiener filter and adaptive filter algorithms namely LMS, NLMS and RLS algorithms in real time environment. All these methods are compared using several parameters like step size, mean and variance of noise, mean square error, signal to noise ratio, speed, no. of. Iterations etc. In the existence work, the authors have compared the performance of the wiener filter \& LMS algorithm in real time environment with sinusoidal input. This paper is extended by comparing different adaptive filter algorithms with the input taken in real time environment. It is observed that RLS algorithm performs noise cancellation better than all other algorithms. But it has high degree of complexity \& cost while NLMS algorithm has moderate speed of performance and it is quietly chosen for several applications.
\end{abstract}

Keywords: Adaptive Filter; Wiener Filter; LMS algorithms; NLMS algorithm; RLS algorithm; Performance Surface; Least Mean Square; Normalized Least Mean Square; Recursive Least Square; Variable Step Size; Performance Comparison; Mean Square Error; Minimum Mean Square Error; Signal to Noise Ratio; Variance; White Gaussian Noise; Sinusoidal Input; Real time Input

\section{Introduction}

Noise cancellation in real time environment is the main schema of this work and the concept is noise signal is estimated using variable filter and the estimated signal is subtracted from another input called as error signal. The error signal is updated by different algorithms of adaptive filter. Adaptive filter has two input signals in that one is reference signal which is used to estimate the noise signal and another input is the mixed signal of real time speech \& noise. The algorithms are selected depending upon the requirement of the user [1]. Wiener filter approach is the one of the oldest method used for noise cancellation and replaced because of its high degree of complexity and low speed [2-3]. In the existing work authors proposed the adaptive filter algorithms like LMS, NLMS and RLS for noise cancellation and their performance is compared, but they have taken the input in sinusoidal environment. This work is extended by replacing the sinusoidal signal with real time signals and the performance of all these filters is compared and the results are presented. 


\section{Objective}

The main objective of this work is to remove the noise that is inherently present in the signal and it is done by using different variable filters like wiener and adaptive filters. It compares the performance of these filters by varying the parameters like complexity, speed, convergence rate, stability, etc.

\section{Related Work}

The authors Yen-Hsiang Chen, Shanq-Jang Ruan and Tom Qi [4], implemented a realtime adaptive wiener filter with two micro phones to reduce noise when noise signals and desired speech are incoming simultaneously. This paper also presents an analysis of different matrix sizes of the wiener filter to enable the possibility of real-time implementation. The performance of the proposed design is measured by as much as 20 $\mathrm{dB}$ noise reduction, and the proposed adaptive wiener matrix update speed achieves a 28.6 $\mathrm{ms} /$ frame, with a matrix size of 200.

The authors H.kaur and R.Talwar [5], instead of computing $W_{\text {opt }}$ as suggested by Wiener-Hopf equation, the coefficients of LMS algorithm are adjusted from sample to sample to minimize the MSE. The LMS algorithm is based on steepest descent algorithm and they also presented the analysis of LMS algorithm for different input samples with different number of iterations and proved that the convergence rate is low for this algorithm.

The authors H. chang and J.Lee [6], described the necessity of NLMS algorithm with variable step size. It has high convergence rate than LMS algorithm due to the variable step size because of input signal amplification problem.

The authors Kaur. H and Talwar.R [7], have compared the performance of different adaptive algorithms like LMS, NLMS, and RLS algorithms for sinusoidal input. Performance is compared for different step size with different number of iterations. Three adaptive filter algorithms have been compared by simulation to achieve high convergence rate and minimum mean square error with noise and different values of $\mu$. Every algorithm works on different methods for noise cancellation and improves system performance.

The authors G.V.P.Chandra Sekhar Yadav and Dr. B. Ananda Krishna [8], proposed the performance of wiener filter and different adaptive filter algorithms like LMS, NLMS and RLS algorithms for noise cancellation in real time environment with recorded speech as the input and different noise signals are added to it and desired signal is estimated by using the adaptive algorithms.

\section{Problem Statement}

The existing work [7] is extended by replacing the input sinusoidal signal with real time signals like recorded speech, noise, etc. and compares the performance of all variable filters with different algorithms.

\section{Wiener Filter}

Wiener filter eliminates the noise in the desired signal by estimating the noise signal from the input reference signal as shown in the Figure 1. 


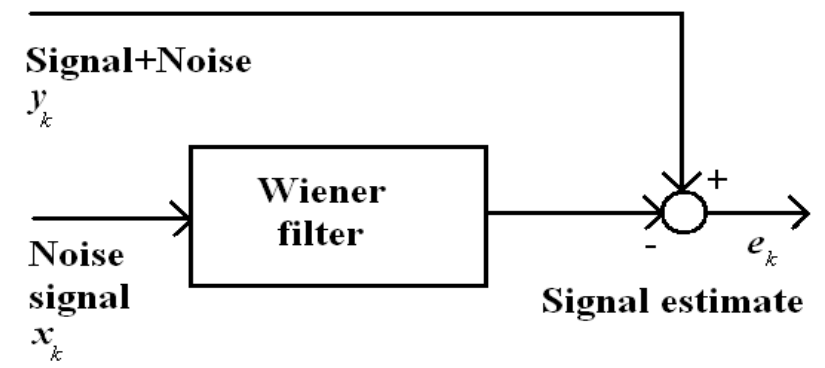

Figure 1. Basic Wiener Filter

Let $x_{k}$ is the noise signal applied as input to the wiener filter and $y_{k}$ is the received signal given to the adder, which adds the output of wiener filter i.e., estimated noise signal $\left(\hat{n}_{k}\right)$ and $y_{k}$ and generates the error signal expressed as

$$
\begin{gathered}
e_{k}=y_{k}-\hat{n}_{k} \\
e_{k}=y_{k}-\sum_{i=0}^{N-1} x_{k-i} w(i)
\end{gathered}
$$

Where $x_{k}$ input signal applied to the wiener filter i.e., reference noise signal and $\mathrm{W}$ is the filter coefficients vector. Error signal $e_{k}$ is minimized by taking the mean square of error.

$$
M S E=\varepsilon\left(e_{k}^{2}\right)=\varepsilon\left(y_{k}^{2}\right)-2 W^{T} R_{Y X}+W R_{X X} W^{T}
$$

MSE is the Mean Square Error of the filter, which decides the nature of output signal obtained. The relation between MSE and filter coefficients W is shown in Figure 2. The error $e_{k}$ is large for the first set of filter coefficients and it is gradually decreased by varying the filter coefficients resembles the bowl shape. The set of filter coefficients for which the error is minimum, is known as Optimum set.

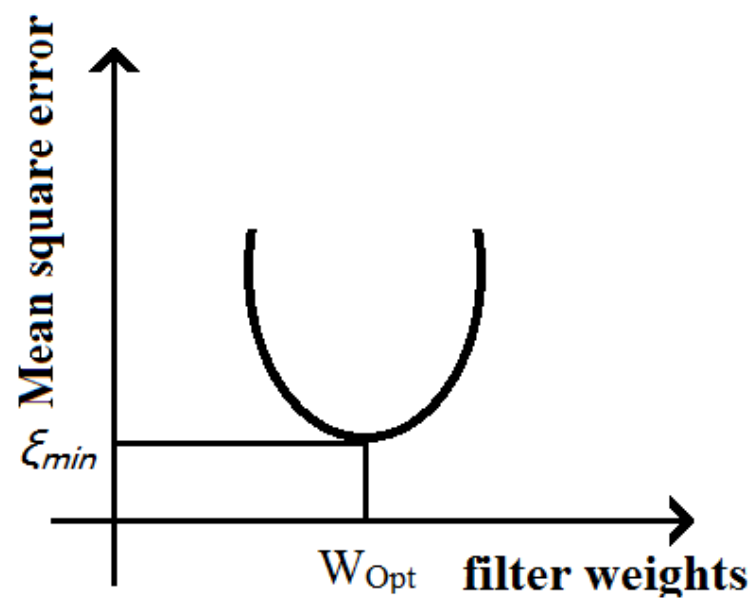

Figure 2. Performance Surface of Wiener Filter

Gradient of the MSE is obtained by calculating differentiation of MSE with respect to filter coefficients. 


$$
\begin{aligned}
\nabla=\frac{d(M S E)}{d(W)} & =\frac{d\left\{\varepsilon\left(y_{k}^{2}\right)-2 W^{T} R_{Y X}+W R_{X X} W^{T}\right\}}{d(W)} \\
\nabla & =0-2 R_{Y X}+2 W R_{X X}
\end{aligned}
$$

Where $R_{Y X}$ is the cross correlation of $x_{k}$ and $y_{k}$ and $R_{X X}$ is the auto correlation of $x_{k}$ Consider $R_{Y X}=\mathrm{P}$ and $R_{X X}=\mathrm{R}$, then gradient of MSE is modified as

$$
\nabla=0-2 P+2 R W
$$

Unique bottom value i.e., minimum value of MSE is obtained by equating gradient value to zero

$$
\begin{gathered}
\nabla=0 \\
-2 P+2 R W=0 \\
2 P=2 R W \\
W_{O p t}=R^{-1} P
\end{gathered}
$$

This is known as Wiener-Hopf Equation [9]. $W_{\text {Opt }}$ is the optimum value of MSE, where the error value is approximately equal to zero, and hence output obtained is the required signal.

A signal is completely recoverable from noise when the spectra of the signal and noise don't overlap each other. If the signal and noise occupies different parts of the frequency spectrum, they can be separated by using either low pass or high pass filter. If the signal and noise has overlap spectra, then it is not possible to completely separate the signal from noise but the effects of noise can be reduced by using wiener filter [10]. Though wiener filter gives the noiseless output it has some practical limitations: In wiener filter, input signals are considered as statistic before processing the signals. It has to calculate both auto correlation and cross correlation $\mathrm{R}$ and $\mathrm{P}$ for each iteration. It involves matrix inversion, matrix multiplication, auto correlation and cross correlation which consumes more time for computing the result for real time applications. Adaptive algorithms are used to achieve this without having to compute $\mathrm{R}$ and $\mathrm{P}$ explicitly or performing a matrix inversion. The solution to Wiener-Hopf equation is the steepest descent algorithm [11].

\section{Adaptive Filtering}

Adaptive filters are the filters which can be modified or adapted or changed according to the requirement to improve filter performance depending on the type of application [12]. Adaptive filter has two steps in the process of filtering. First it filters the input signal and generates the filtered signal, after that it modifies the coefficients of filtered signal according to different algorithms, until it gets the required signal.

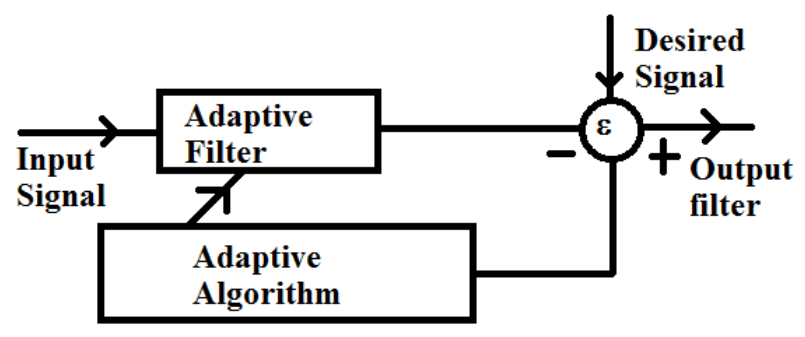

Figure 3. Adaptive Filter 
Figure 3 shows adaptive filter process, which has two parts i.e., filter and adaptive algorithm. Filter generates the estimated signal of the input reference signal, and it compares the estimated signal with the desired signal which is the mixed signal of speech and noise. The output of the summer is called error signal. This error signal is modified according to the specified adaptive algorithm and produces the estimated signal. This process is continued until the exact noise signal is estimated. Adaptive algorithms used in the feedback path are Least Mean Square (LMS) algorithm, Normalized Least Mean Square (NLMS) algorithm and Recursive Least Square (RLS) algorithm which are discussed in the following sections.

In the basic form, the wiener theory assumes that signals are stationary. However in adaptive filters, the coefficients are periodically regulated for every $\mathrm{N}$ samples and the filter adapts itself to the characteristics of the signal. Adaptive filter provides alternate solution to the formulation of wiener filter limitations [13]. Adaptive filters are used in the following cases:

- When it is necessary for the filter characteristics to be variable, adapted to changing conditions.

- When there is spectral overlap between signal and noise.

- If the band occupied by the noise is unknown or varies with time.

- The use of conventional filters in the above cases would lead to unacceptable distortion of the desired signal.

\subsection{Adaptive Filter as Noise Canceller}

Adaptive filter has several applications out of those noise cancellation is one of the major application used in real time environment efficiently.

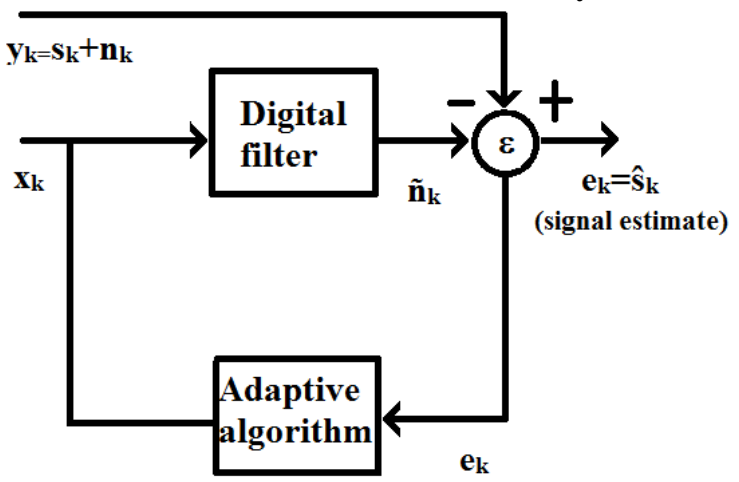

Figure 4. Adaptive filter as noise canceller

Figure 4 shows the block diagram of adaptive filter as noise canceller. Where $y_{k}$ and $x_{k}$ are the inputs to the digital filter and summer respectively. $x_{k}$ is the reference signal to be estimated with the help of digital filter via specified algorithm. $y_{k}$ is the combination of desired signal $s_{k}$ and noise signal $n_{k}$ which is correlated with reference signal $x_{k}$ while uncorrelated with other desired signal $s_{k}$ [14]. The estimated signal $\hat{n}_{k}$ is given to adder/sub tractor and also the combined signal $y_{k}$ is given, the output of adder/sub tractor is error signal which is then updated with the help of adaptive algorithm until estimated signal is approximated to the noise signal in $y_{k}$.

$$
\hat{s}_{k}=y_{k}-\hat{n}_{k}=s_{k}+n_{k}-\hat{n}_{k}
$$

Output of adder/sub tractor can act as the estimate of desired signal $\hat{s}_{k}$ and an error signal $e_{k}$ which can be used to update the estimated signal. 


\section{Algorithms of Adaptive Filter}

Adaptive filters utilizes adaptive algorithms to update the signal characteristics i.e., coefficients in the feedback path. There are three different algorithms for updating the coefficients namely Least Mean Square (LMS) algorithm, Normalized Least Mean Square (NLMS) algorithm and Recursive Least Square (RLS) algorithm.

\subsection{Least Mean Square (LMS) Algorithm}

LMS algorithm is one of the simplest algorithm that is used in adaptive applications. Instead of calculating optimum value $W_{O p t}$ as suggested by Wiener-Hopf equation, LMS algorithm provides solution by updating filter coefficients sample by sample to minimize Mean Square Error. The standard LMS algorithm performs the following operations to update the coefficients of an adaptive filter:

- Calculates the output signal from the adaptive filter.

- Calculates the error signal by using the following equation.

$$
e_{k}=y_{k}-\widehat{n_{k}}=y_{k}-\sum_{i=0}^{N-1} w(i) \cdot x_{k-i}
$$

- Updates the filter coefficients by using the following equation.

$$
W_{k+1}=W_{k}+2 \mu e_{k} x_{k}
$$

Where $\mu$ is the step size of the adaptive filter, $W_{k}$ is the filter coefficients vector, and $x_{k}$ is the filter input vector. $\mu$ is the correction factor applied to the error signal which corrects the signal to get desired estimation.

The LMS algorithm is based on the steepest descent algorithm where the weight vector is updated from sample to sample is shown in Figure 5 is as follows:

$$
W_{k+1}=W_{k}-\mu \nabla_{k}
$$

Where $W_{k}$ and $\nabla_{k}$ are the weight and the true gradient vectors respectively at the $k^{t h}$ sampling instant. $\mu$ controls the size of incremental correction applied to the adaptive filter from one iteration to the next.



Figure 5. Adaptive Filter using LMS Algorithm

The steepest descent algorithm in the above equation still requires knowledge of $\mathrm{R}$ and P. since $\nabla_{k}$ is obtained by evaluating the equation.

$$
\nabla=\frac{d(M S E)}{d w}=0-2 P+2 R W
$$


The LMS algorithm is a practical method of obtaining estimates of the filter weights $W_{k}$ in real time without matrix inversion in the equation $W_{o p t}=R^{-1} P$ or the direct computation of the auto correlation and cross correlation [15].

$$
\nabla=-2 P+2 R W
$$

In the LMS algorithm, instantaneous estimates are used for $\nabla$. Thus

$$
\begin{gathered}
\nabla_{k}=-2 P+2 R W \\
\nabla_{k}=-2 x_{k} y_{k}+2 x_{k} x_{k}^{T} W_{k} \\
\nabla_{k}=-2 x_{k}\left(y_{k}-x_{k}^{T} W_{k}\right) \\
\nabla_{k}=-2 x_{k} e_{k}
\end{gathered}
$$

Where

$$
e_{k}=y_{k}-x_{k}^{T} W_{k}
$$

Replace the value of $\nabla_{k}$ in steepest descent algorithm then

$$
\begin{gathered}
W_{k+1}=W_{k}-\mu \nabla_{k} \\
W_{k+1}=W_{k}-\mu\left(-2 x_{k} e_{k}\right) \\
W_{k+1}=W_{k}+2 \mu e_{k} x_{k}
\end{gathered}
$$

Clearly, the LMS algorithm above doesn't require prior knowledge of the signal statistics (that is the correlations $\mathrm{R}$ and $\mathrm{P}$ ), but instead uses their instantaneous estimates as shown above [16].

The weights obtained by the LMS algorithm are only estimates, but these estimates improve gradually with time as weights are adjusted and the filter learns the characteristics of the signals. Eventually, the weights converge [17].

The condition of convergence is

$$
0<\mu>2 / \lambda_{\operatorname{Max}}
$$

Where $\lambda_{\operatorname{Max}}$ is the maximum Eigen value of the input data covariance matrix.

In general $\mathrm{W}_{\mathrm{k}}$ never reaches the theoretical optimum (the wiener solution), but fluctuates as shown in Figure 6.

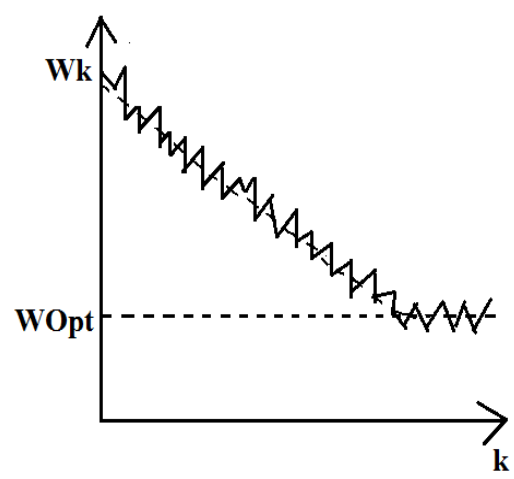

Figure 6. An illustration of the Variations in the Filter Weights 
The simplicity of the LMS algorithm and ease of implementation, make the algorithm of first choice in many real-time systems. The LMS algorithm requires approximately $2 \mathrm{~N}+1$ multiplications and $2 \mathrm{~N}$ addition for each new set of input and output samples. In general any signal processing operations involves large no of multiply and accumulate arithmetic operations, because of direct implementation LMS algorithm is more attractive.

\subsection{Normalized Least Mean Square (NLMS) Algorithm}

NLMS algorithm provides solution to the drawback of LMS algorithm. LMS algorithm has drawback of input sampling problem. This problem occurs when the amplitude of noise signal is too large, called as gradient noise amplification [18]. NLMS algorithm is similar to that of LMS algorithm except it has variable step size instead of constant one. It provides solution to noise amplification problem by providing variable step size as shown below.

Where

$$
\begin{gathered}
W_{k+1}=W_{k}+\mu_{k} x_{k} e_{k} \\
\mu_{k}=\frac{\mu}{x_{k}^{T} x_{k}} ; 0<\mu<2
\end{gathered}
$$

$\mu$ is the adaption constant for NLMS algorithm and it is between 0 and 2 to optimize the convergence rate.

In the above equation, step size is normalized with the power of input signal $x_{k}$. Step size is used to increase the convergence speed, so that its performance gets increased. Adaptive filter using NLMS algorithm as updating algorithm in feedback is shown in Figure 7. NLMS algorithm provides solution to the gradient noise amplification problem, but if the amplitude value of input signal is too small i.e., zero then variable step size value become infinite, then problem arises [19].

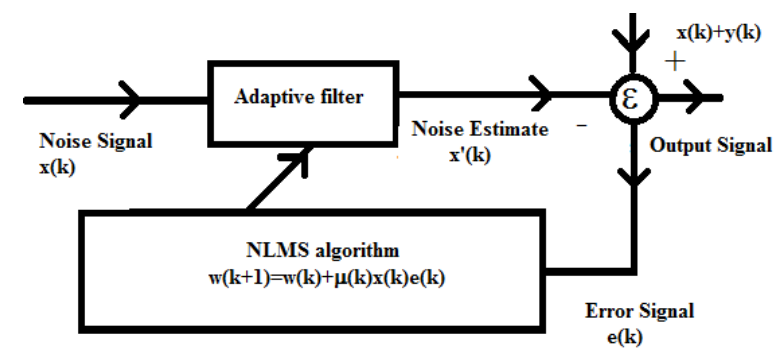

Figure 7. Adaptive Filter using NLMS Algorithm

To solve this problem there should be regularization. Hence the updating equation is modified as

$$
W_{k+1}=W_{k}+\frac{\mu}{\delta+x_{k}^{T} x_{k}} x_{k} e_{k}
$$

Where ' $\delta$ ' is the regularized value always less than 1 .

\subsection{Recursive Least Square (RLS) Algorithm}

Recursive Least Squares algorithm is designed to overcome the drawback of LMS and NLMS algorithms i.e., high convergence rate. RLS algorithm is based on deterministic philosophy while other algorithms are based on stochastic philosophy [20]. RLS algorithm is the fastest algorithm which gives results faster than any other algorithm, but this fastness adds high degree of complexity to algorithm hence it has high cost. RLS algorithm is best suitable for real time applications. As mean square value is reduced the stability of the system is getting unstable, so stability problem arises in RLS algorithm due to high convergence rate [21]. The concept of RLS algorithm is to minimize the cost function by proper filter coefficient value and updating the coefficients sample by sample 
as shown below. Error signal and updating algorithm (RLS) are given in feedback path to correct the signal according to the correction factor provided in the algorithm [22].

The error signal is the output of adder/sub tractor and it is given by

$$
e_{k}=y_{k}-\hat{n}_{k}
$$

Where $y_{k}$ is the desired signal and $\hat{n}_{k}$ is the estimate of reference signal which is correlated with the noise signal in the desire signal. $\hat{n}_{k}$ is the output of adaptive filter.

The cost function ' $\mathrm{C}$ ' depends on the error function, and it has to minimize the error function $e_{k}$ which is dependent on the filter coefficients.

$$
C\left(W_{k}\right)=\sum_{i=0}^{k} \lambda^{n-i} e_{k}^{2}
$$

Where $\lambda$ is the forgetting factor and is very $\dot{c}$ close to and less than 1 i.e., $0<\lambda \leq 1$.

The cost function is minimized by taking the partial derivatives for all entries of the coefficient vector $W_{k}$ and equates the results to zero.

Finally the RLS algorithm for an $p^{\text {th }}$ order filter can be

$$
\begin{gathered}
\alpha_{k}=y_{k}-x_{k}^{T} W_{k-1} \\
g_{k}=p_{k-1} x_{k}^{*}\left\{\lambda+x_{k}^{T} p_{k-1} x_{k}^{*}\right\}^{-1} \\
p_{k}=\lambda^{-1} p_{k-1}-g_{k} x_{k}^{T} \lambda^{-1} p_{k-1} \\
W_{k}=W_{k-1}+\alpha_{k} g_{k}
\end{gathered}
$$

Where $\mathrm{p}=$ filter order

$\lambda=$ forgetting factor

$\delta=$ value to initialize $p_{0}$

$W_{k}=0$

$p_{0}=\delta^{-1} I$ Where I is the identity matrix of rank $\mathrm{p}+1$

\section{Simulation Results}

The proposed work has been implemented and simulated using MATLAB, in real time environment by considering input as recorded speech and recorded noise. Also random noise is taken as input in the place of recorded noise and the performance is compared for different mean and variance of random noise with different correction factors or step sizes for different number of iterations.

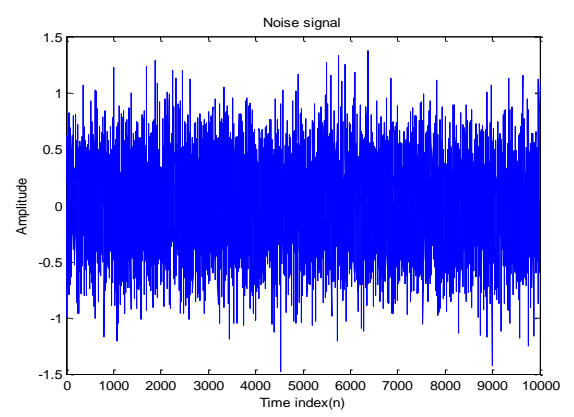

Figure 8. Random Noise Signal 


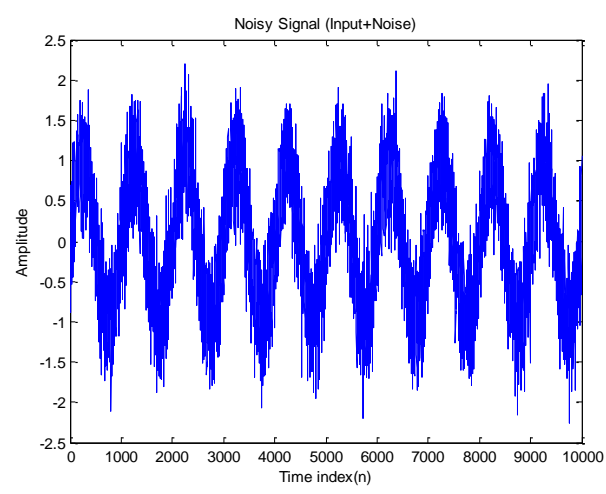

Figure 9. Noisy Signal

Figure 8, 9 and 10 shows the inputs and output of the filters respectively. One of the inputs taken is in sinusoidal environment and other input which is given to adaptive filter is random noise signal as shown in Figure 8 and 9 respectively.

From Figure 10 it is clear that the output of all variable filters like wiener filter, LMS, NLMS and RLS algorithms produces sinusoidal signal as their output, and RLS algorithm removes noise accurately than any other algorithm at high convergence rate and high degree of complexity. All these three figures are related to the existing work proposed by Kaur. $\mathrm{H}$ and Talwar. R [7].

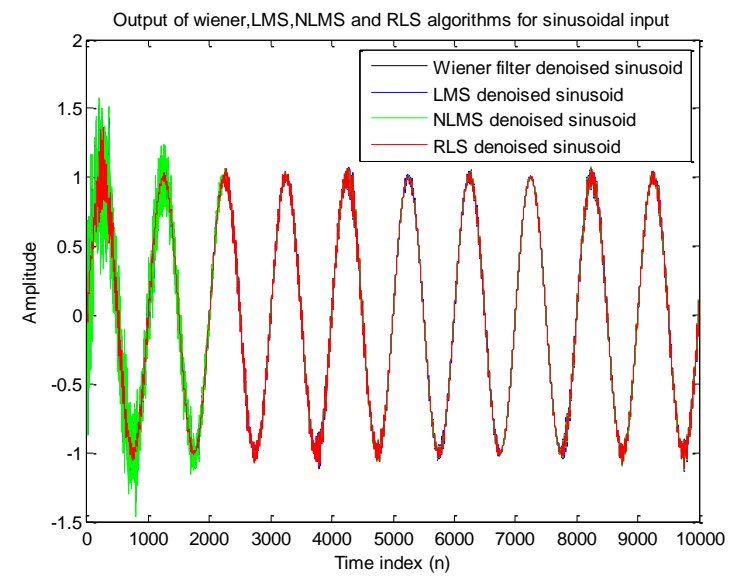

Figure 10. Output of Different Filters in Sinusoidal Environment

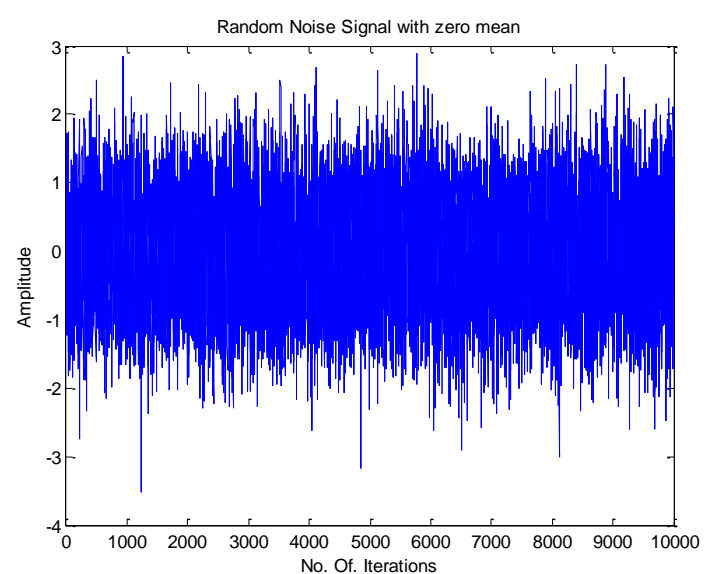

Figure 11. White Gaussian Noise Signal with Zero Mean 




Figure 12. Noisy Signal with White Gaussian Noise

Figure 11, 12 shows the inputs of the proposed work, which are given to adaptive filter and adder/ sub tractor respectively. Figure 11 shows the white Gaussian noise signal with zero mean and Figure 12 shows the combination of recorded speech signal with the help of micro phone using MAT LAB software and white Gaussian noise signal shown in Figure 11.

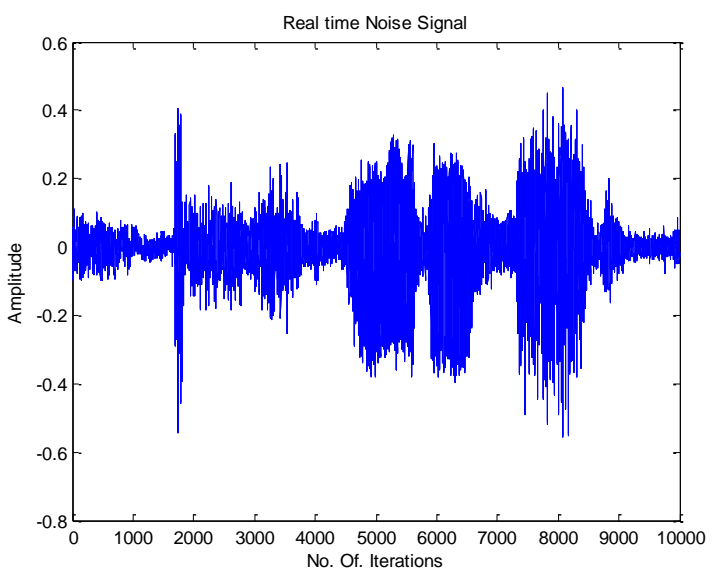

Figure 13. Real Time Recorded Noise Signal

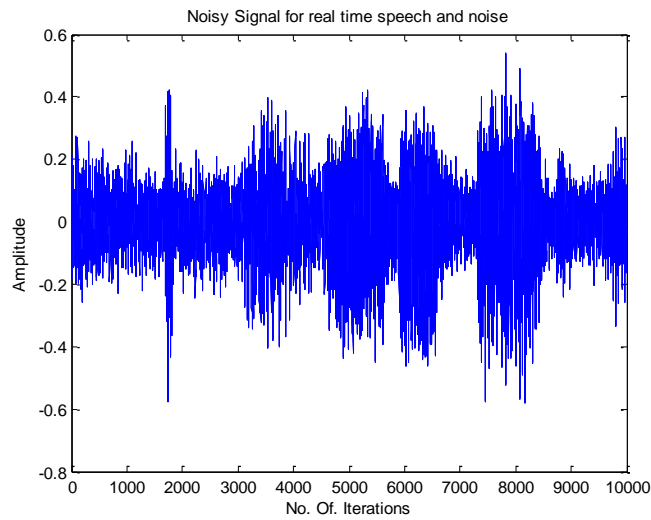

Figure 14. Noisy Signal for Real Time Inputs 
Noise signal is replaced with recorded noise signal and is shown in Figure 13. And this recorded noise signal is added with the recorded speech signal and the combination of mixed signal is shown is Figure 14.

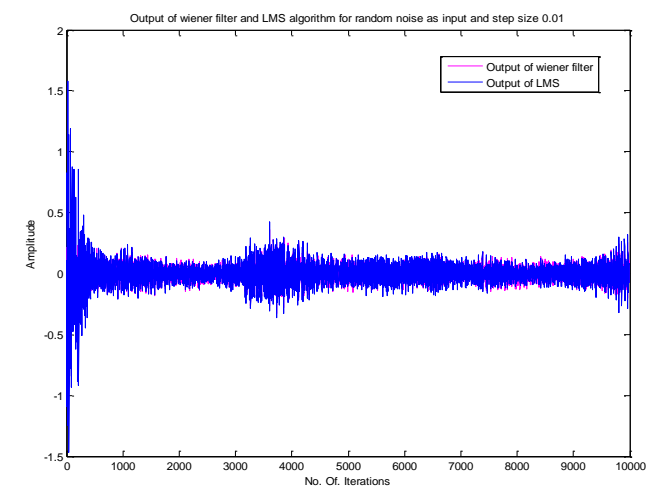

Figure 15. Output of Wiener and LMS Algorithm for Random Noise as Input and $\mu=0.01$

All of these input signals are given and responses of all the variable filters like wiener filter, LMS, NLMS and RLS algorithms are plotted.

Figures 15, 16, 17 and 18 shows the output of wiener filter and LMS, output of NLMS and RLS, output of all these variable filters and Mean Square Error of all these variable filters respectively by considering white Gaussian noise signal as the input with step size 0.01 .

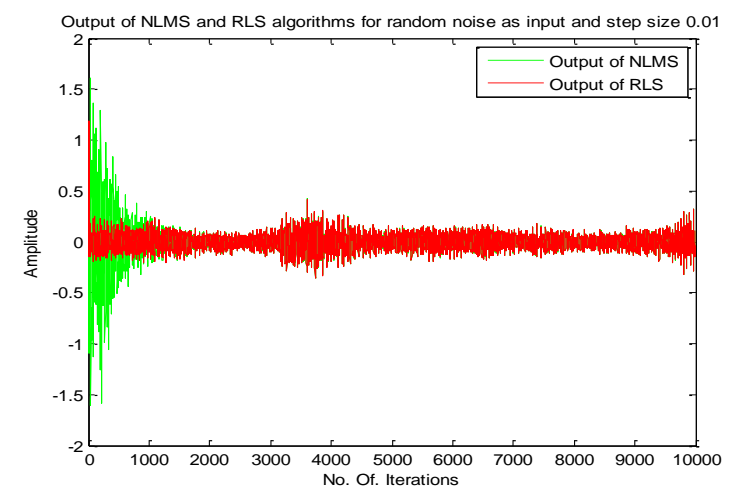

Figure 16. Output of NLMS and RLS Algorithm for Random Noise as Input and $\mu=0.01$

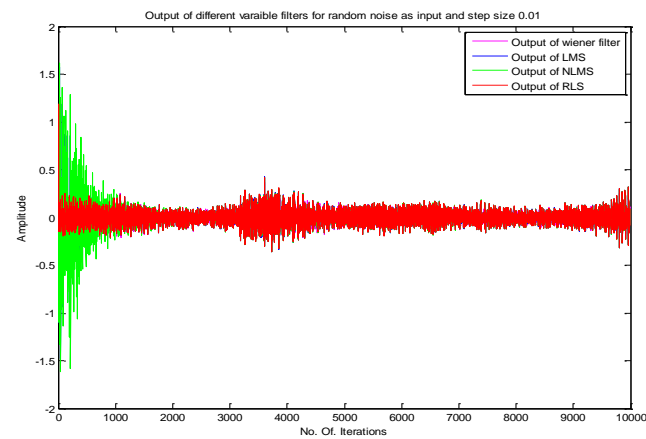

Figure 17. Output of Wiener, LMS, NLMS and RLS Algorithms for Random Noise as input and $\mu=0.01$ 
By observing all these responses RLS algorithm provides better results with fast response but has less stability than other algorithms. NLMS algorithm provides trade-off between LMS algorithm and RLS algorithm.

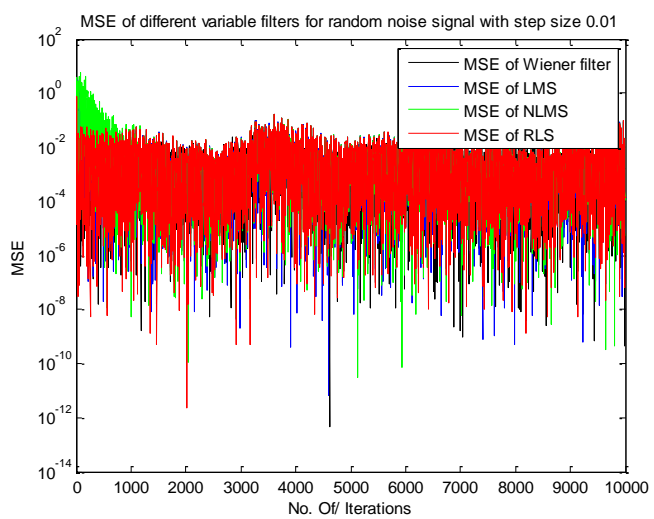

Figure 18. MSE of wiener, LMS, NLMS and RLS Algorithms for Random Noise as Input and $\mu=0.01$

Figure 18 shows the MSE of variable filters for white Gaussian noise as input with step size of 0.01 , form the plot it is clear that RLS algorithm removes noise efficiently than other algorithms, whereas NLMS algorithm removes noise but less than that of RLS algorithm. Similarly LMS algorithm also removes noise but less than NLMS and RLS algorithms. MSE should be as low as possible in order to get quality response.

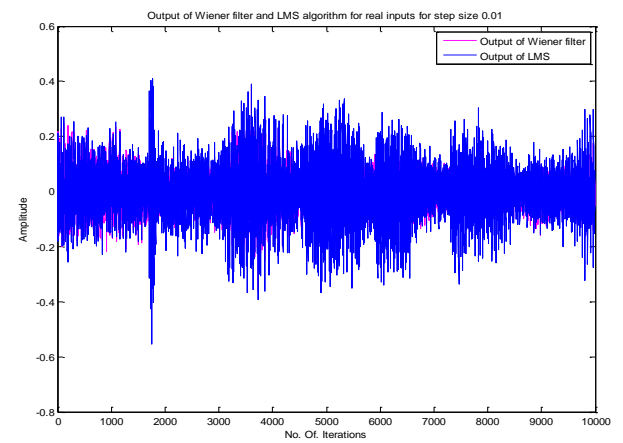

Figure 19. Output of Wiener and LMS Algorithm for Real Time Input and $\mu=0.01$

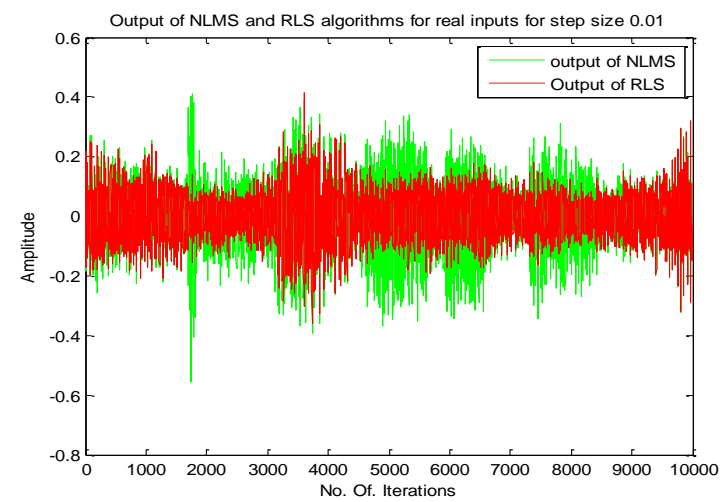

Figure 20. Output of NLMS and RLS Algorithm for Real Time Input and $\mu=0.01$ 


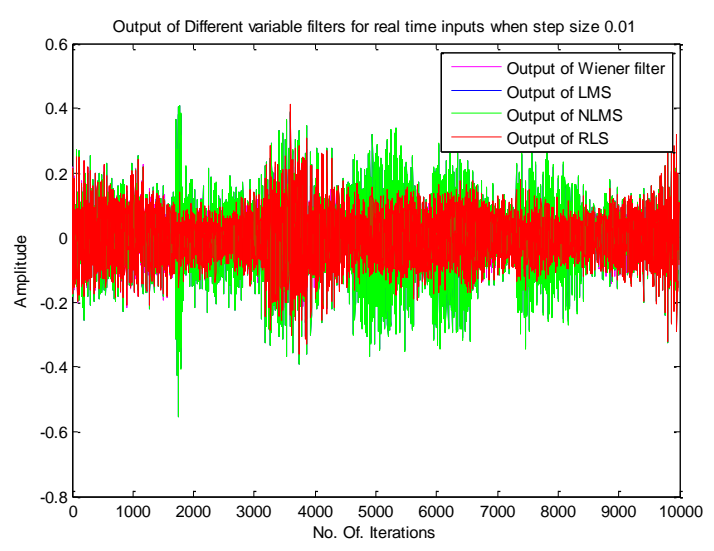

Figure 21. Output of Wiener, LMS, NLMS and RLS Algorithm for Real Time Input and $\mu=0.01$

Figure 19, 20 and 21 shows the output of wiener filter and LMS algorithm, output of NLMS and RLS algorithm, output of all these variable filters in real time environment for step size of 0.01 respectively.

From observation RLS algorithm provides better response than other algorithms, and this facility is available with high complexity and cost. MSE of RLS algorithm is low than all other algorithms, hence it is less stable.

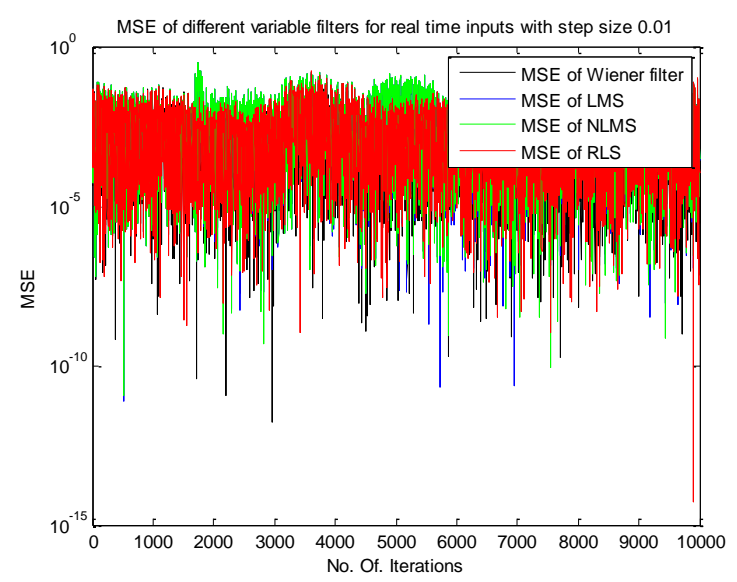

Figure 22. MSE of Wiener, LMS, NLMS and RLS Algorithm for Real Time Input and $\mu=0.01$

Figure 22 shows the MSE of all variable filters in real time environment for step size 0.01 . MSE is the Mean Square Error, which decides the quality of the response obtained. As MSE is low then quality response obtained is high and for low quality response systems MSE should be high. For getting qualified output one should have to minimize MSE as low as possible. 


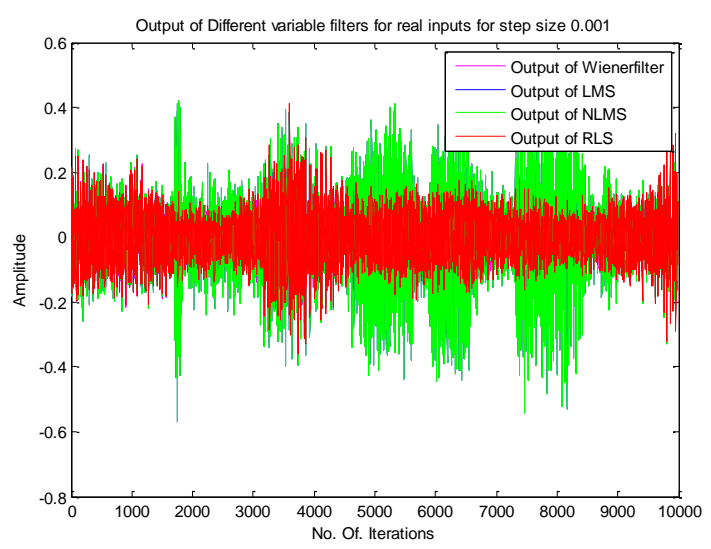

Figure 23. Output of Wiener, LMS, NLMS and RLS Algorithm for Real Time Input and $\mu=0.001$

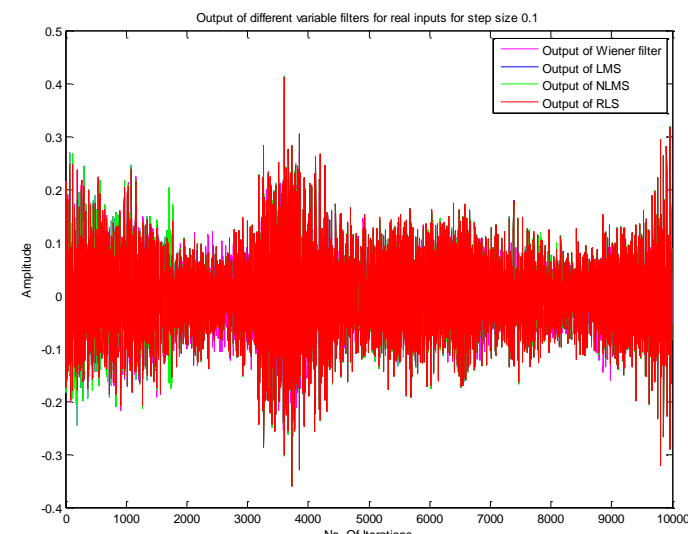

Figure 24. Output of Wiener, LMS, NLMS and RLS Algorithm for Real Time Input and $\mu=0.1$

Figure 23 and 24 shows the output of different variable filters in real time environment for 0.001 and 0.1 step sizes respectively.

Correction factor or step size is the value which defines the amount of correction applied to the filter through feedback algorithm. As the correction factor is too high then the speed of operation is fast, but it has poor filtering nature and if correction factor is too low then response is slow, but has high filtering nature, hence compromised value is taken for step size in order to get good speed and filtering.

Complexity means number of computations i.e., adders, multiplications etc., to get fast response in addition to step size complexity is also considered and it is as low as possible. Table 1 shows the comparison of number of additions, multiplications and stability of adaptive algorithms. LMS algorithm provides low complexity than other algorithms, but has low convergence rate while RLS algorithm has high complexity, but it has high convergence rate. Hence compromised NLMS algorithm is the best choice for different applications. 
Table 1. Comparison of No. of Additions, Multiplications and Stability of Adaptive Algorithms

\begin{tabular}{|c|c|c|c|c|c|c|c|c|c|}
\hline \multirow{2}{*}{$\begin{array}{c}\text { No. of } \\
\text { Iterations }\end{array}$} & \multicolumn{3}{|c|}{ No. of Additions } & \multicolumn{3}{|c|}{ No. of Multiplications } & \multicolumn{3}{|c|}{ Stability } \\
\hline & LMS & NLMS & RLS & LMS & NLMS & RLS & LMS & NLMS & RLS \\
\hline 1 & 2 & 3 & 4 & 3 & 4 & 7 & \multirow{3}{*}{ 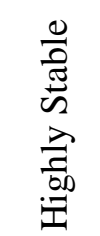 } & \multirow{3}{*}{$\begin{array}{l}\frac{0}{a} \\
\frac{\pi}{n}\end{array}$} & \multirow{3}{*}{ 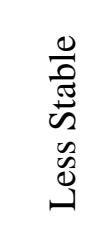 } \\
\hline 2 & 4 & 6 & 10 & 5 & 7 & 15 & & & \\
\hline 10 & 20 & 30 & 130 & 21 & 31 & 151 & & & \\
\hline
\end{tabular}

Stability is the factor that decides the nature of the system that produces output. As long as MSE is reduced stability of the system is getting reduced. For LMS algorithm MSE is high than NLMS and RLS, but it is highly stable. RLS algorithm has low MSE, so it is less stable. Hence, here in this scenario also NLMS algorithm is the best choice for compromised MSE and stability.

Table 2. Comparison of All Variable Filters in Real Time Environment

\begin{tabular}{|c|c|c|c|}
\hline Algorithm & Speed & Convergence rate & MSE \\
\hline Wiener & Low & - & Moderate \\
\hline LMS & Medium & Low & High \\
\hline NLMS & High & Moderate & Low \\
\hline RLS & Very high & High & Very low \\
\hline
\end{tabular}

Table 2 shows the performance comparison of all variable filters in the area of speed, convergence rate and MSE. Speed is the factor that decides the characteristics of the system; convergence rate is the factor that decides the speed of the response and quality of filtering. Convergence rate is the correction factor applied back to the filter through feedback. MSE is also the factor that describes the quality of the response.

Table 3 shows Mean Square Value of all variable filters like wiener filter, LMS algorithm, NLMS algorithm and RLS algorithm for different step size values.

Table 3. Comparison of MSE of All Variable Filters in Real Time Environment

\begin{tabular}{|c|c|c|c|c|}
\hline \multirow{2}{*}{ Step Size } & \multicolumn{4}{|c|}{ Mean Square Error } \\
\cline { 2 - 5 } & Wiener & LMS & NLMS & RLS \\
\hline 0.01 & 0.0170 & 0.0170 & 0.0172 & 0.0151 \\
\hline 0.1 & 0.0170 & 0.0152 & 0.0152 & 0.0175 \\
\hline
\end{tabular}




\begin{tabular}{|l|l|l|l|l|}
\hline 0.001 & 0.0170 & 0.0214 & 0.0213 & 0.0308 \\
\hline 0.5 & 0.0170 & 0.0155 & 0.0155 & 0.0046 \\
\hline 0.05 & 0.0170 & 0.0151 & 0.0154 & 0.0076 \\
\hline
\end{tabular}

From the observation it is clear that step size didn't show any effect on wiener filter output hence its MSE value remains unchanged and for different step size values different values are obtained. Depending upon the requirement one should go with the proper step size value.

The chart shows the SNR comparison of different variable filters for different iterations. SNR is the Signal to noise ratio which is used to measure the percentage of noise reduced from input to output. Improvement in SNR resembles the improvement of signal at the output. As long as iterations increase SNR gets increases. RLS algorithm shows improvement in SNR than any other algorithms shown in chart below.

$\square$ SNR at Input $\square$ SNR after Wiener Filter $\square$ SNR after LMS $\square$ SNR after NLMS $\square$ SNR after RLS



\section{No. Of. Iterations \\ Comparison SNR of Different Variable Filters for Different Number of Iterations}

\section{Conclusion and Future Work}

From the results analysis, performance of variable filter algorithms are concluded as wiener filter is the ancient method that removes noise from the signal and it is suitable for stationary environment and it has large number of calculations. Since the proposed work is implemented in real time environment there is a necessity of moving to another filter called adaptive filter which is suitable for non-stationary environment. Adaptive filter has three different algorithms to update the filter coefficients. Out of these LMS algorithm is the simplest and best suitable algorithm for low speed applications and it has input amplification effect. NLMS algorithm is similar to that of LMS with moderate speed of operation. Furthermore to increase the speed RLS algorithm is suited and it works in deterministic environment but it has high degree of complexity and more cost. NLMS algorithm provides trade-off between convergence rate and complexity; hence it is the choice of several applications. Depending upon the type of application either of these algorithms is chosen. If one requires noise cancellation application for low cost then LMS algorithm is the preferred similarly if application required with high speed of operation 
RLS algorithm is preferred where it requires "No Matter of Cost" as the tag line. The entire work is done in real environment like recorded speech, recorded noise, watermark noise, wind noise etc along with sinusoidal noise. As a future work different noise sources like watermark noise, wind noise etc can be removed in real time environment.

\section{Acknowledgments}

The authors wish to thank to everyone who helped them regarding the work and analysis.

\section{References}

[1] P. S. R. Diniz, “Adaptive Filtering: Algorithms and Practical Implementations”, ISBN 978-0-387-312743, Kluwer Academic Publisher 2008 Springer Science + Business Media, LLC, pp. 77-195.

[2] G. V. P. Chandra Sekhar Yadav and Dr. B. Ananda Krishna and Dr. M. Kamaraju, "Performance of wiener filter and adaptive filter for noise cancellation in real time environment", International journal of computer applications (0975-887), vol. 97, no. 15, (2014) July.

[3] K. A. Lee, W. S. Gan, and S. M. Kuo, "Subband Adaptive Filtering: Theory and Implementation", Hoboken, NJ: Wiley, (2009).

[4] Yen-Hsiang chen, Shanq-Jang Ruan and Tom Qi, "An Automotive Application of real time adaptive wiener filter for noise cancellation in a car environment", IEEE,2012,4673-2193.

[5] H. Kaur and R. Talwar, "Performance and Convergence Analysis of LMS Algorithm", IEEE ICCIC, (2012) Dec.

[6] H. Chang and J. Lee, "A Variable Step Size NLMS Algorithm and its Performance Analysis", IEEE Trans. Signal Process, vol. 60, no. 4, (2012) April.

[7] H. Kaur and R. Talwar, "Performance comparison of adaptive algorithms for noise cancellation", Engineering trends in communication, C2SPCA, (2013).

[8] G. V. P. Chandra Sekhar Yadav and Dr. B. Ananda Krishna, "Study of different adaptive filter algorithms for noise cancellation in real time environment", International journal of computer applications (0975-887), vol. 96, no. 10, (2014) June.

[9] M. A. Noor, "Wiener-Hopf Equations and variational Inequalities", Journal of Optimization Theory and Applications, vol. 79, no. 01, (1993) Oct.

[10] M. A. Abd El-Fattah, M.I. Dessouky, S.M. Diab and F.E. Abd EI-samie, "Adaptive Wiener Filtering Approach for Speech Enhancement”, Ubiquitous Computing and Communication Journal, vol. 03, no. 02, (2008) April.

[11] B. Widrow and S. D. Stearns, "Adaptive Signal Processing, Englewood Cliffs", NJ: Prentice- Hall, (1985).

[12] S. Haykin, "Adaptive Filter Theory", Fourth edition, Upper saddle River, NJ: Prentice -Hall, (2002)

[13] B. Widrow, J. R. Glover, "Adaptive Noise Cancelling: Principles and Applications”, IEEE Proceedings, Vol-63, No.12, (1975) Dec.

[14] J. C. M. Bermudez, "Adaptive Filtering - Theory and Applications", IRIT - INP-ENSEEIHT, Toulouse, (2011) May.

[15] C. Gabriela and M. Sarachin, "Echo Cancellation Using LMS Algorithm", U.P.B Sci Bull., Series C, vol. 71, no. 4, (2009).

[16] J. Gorriz and J. Ramrez, "A Novel LMS Algorithm Applied to Adaptive Noise Cancellation", IEEE Signal Process Letters, vol. 16, no. 1, (2009) Jan.

[17] D.T.M. Slock, "On the convergence behavior of the LMS and the normalized LMS algorithms", IEEE Transactions on Signal Processing, 1993, vol. 41, no. 9, pp. 2811-2825.

[18] H. Chang and J. Lee, "Performance Comparison of Variable Step Size NLMS Algorithms", World Congress on Eng. And Computer Science, vol. 1, no. 4, (2009) Oct.

[19] C. Paleologu, J. Benesty and S. L. Christopher, "Variable Step Size NLMS Algorithm designed for Echo Cancellations", IEEE 2009.

[20] R. K. Thenua, S. K. Agarwal, "Simulation and performance analysis of adaptive filter in noise cancellation", International Journal of Engineering Science and Technology, vol. 2, No. 9, (2010), pp. 4373-4378.

[21] Y.-S. Lau, Z. M. Hussian and Richard Harris, "Performance of Adaptive Filtering Algorithms: A Comparative Study", Australian Telecommunications, Networks and Applications Conference (ATNAC), Melbourne, (2003).

[22] Y. He, H. He, L. Li, Y. Wu and H. Pan, "The Applications and Simulation of Adaptive Filter in Noise Canceling”, 2008 International Conference on Computer Science and Software Engineering, (2008), vol. 4 , pp. $1-4$. 


\section{Authors}

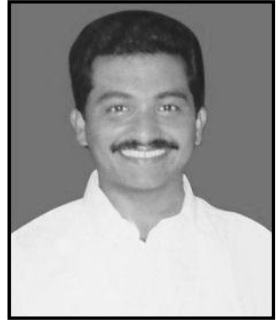

Dr.Ananda Krishna $B$ graduated from Madras University in Electronics and Communication Engineering during the year 1999. He obtained his Master degree in Digital Electronics \& Advanced Communication from Manipal Institute of Technology, Manipal and Ph.D in the area of Improving Quality of service through Secured Routing in Ad Hoc Networks at Jawaharlal Nehru Technological University, Hyderabad. Presently he is working as a Professor in the department of ECE at Sri Mittapalli College of Engineering College, Guntur Dt., AP.

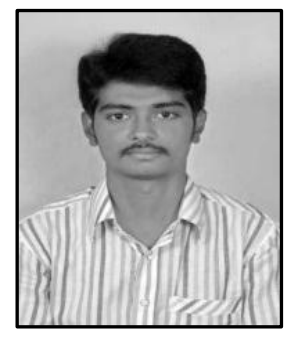

Mr. G.V.P Chandra Sekhar Yadav graduated from DVR \& Dr HS MIC College of Technology, affiliated to JNTU Kakinada in Electronics and Communication Engineering. He obtained his Master degree in Digital Electronics \& Communication Systems from Gudlavalleru Engineering College, Gudlavalleru and his area of interest is Wireless Communication and Signal processing. Presently he is working as an Assistant Professor in the department of ECE at DVR \& Dr HS MIC College of Technology, Kanchikacherla, Krishna Dt., AP. 
International Journal of Signal Processing, Image Processing and Pattern Recognition Vol.9, No.2 (2016) 